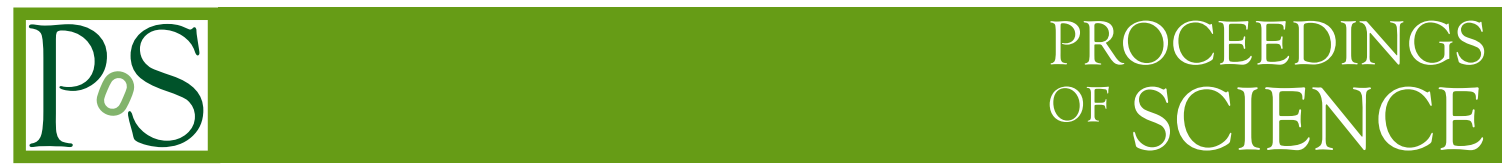

\title{
ATLAS, Play! \\ Teaching particle physics through educational games
}

\section{Sascha Mehlhase}

on behalf of the ATLAS Collaboration

Ludwig-Maximilians-Universität München

E-mail: sascha.mehlhase@cern.ch

Educational games and activities have proven benefits for achieving learning objectives. They motivate competition, provide immediate rewards for success, and encourage collaborative, problembased learning - all while framed as a fun activity, rather than laborious task. An overview of ATLAS educational games developed by members of the ATLAS Collaboration is given, including: 'Making a Splash', a water-balloon game teaching the physics of colliders; 'Proton Cookies', explaining the constituents of a proton through baked goods; 'Build Your Own Particle Detector', using stackable plastic bricks to talk about particle detectors and physics ; and 'Particle Twister', teaching the Standard Model through the game of Twister. Use cases of these games are described and user feedback is presented.

40th International Conference on High Energy physics - ICHEP2020

July 28 - August 6, 2020

Prague, Czech Republic (virtual meeting) 
One of the goals in science communication, as pursued by the ATLAS Collaboration [1, 2], is to nurture support for science in general and the field - particle physics - in particular, as well as to target audience groups as wide and diverse as possible. Sometimes, if not often, it is difficult to leave the filter bubble and reach new audiences using traditional tools of science communication. While many approaches, like setting up science pavilions, exhibitions and/or shows at music festivals or in shopping malls, are being pursued, this contribution focusses on a few activities that take a playful approach at both creating some attention and transferring knowledge to possibly/hopefully new target audiences.

Educational games and activities have proven benefits for achieving learning objectives. They motivate competition, provide immediate rewards for success, and encourage collaborative, problem-based learning for a wide range of age groups and (to some extent) independent of their background. Last but not least they are also fun for scientists and hence allow them to share their enthusiasm in a very informal environment.

The following sections will highlight a selection of activities, list what is required and available to run them, suggest possible topics the audience could be educated in while involved, and present some impressions and qualitative feedback collected during previous events organised by the ATLAS Collaboration.

\section{Making a Splash}

In 'Making a Splash' - an idea originally developed by Ewan Hill and colleagues at TRIUMF - visitors are invited to throw water-balloons at fixed targets or make them collide in mid air. While smashing water-balloon protons from different heights into a pool looking like a particle-physics detector, visitors can learn about energy and momentum conservation, why we need high-energy collisions at the Large Hadron Collider (LHC), the challenge of actually making particles collide, and more.

Our setup for the activity included quite a few water balloons, a plastic kids pool turned into a fixed-target experiment by adding a schematic detector design printed on a plastic sheet, as well as a set of posters to help explain some basic concepts [3-5].

'Making a Splash' - as visible in some impressions in Figure 1 - is a great (good-weather) outdoors activity to get people's attention and to involve them into a discussion about some of the most basic principles in accelerator and particle physics as well as about particle-physics detectors.

\section{Proton Cookies}

Not only love goes through the stomach, also physics can. In 'Proton Cookies' - originally developed by Katharine Leney and Katy Tschann-Grimm of @PhysicsCakes [6] - visitors are invited to build a 'proton' using candy quarks and gluon icing. While 'building' the proton (or any other hadron), visitors can learn about quarks, gluons, the strong interaction and binding energy; why and where protons matter at the LHC; what happens when we collide hadrons; and more.

To open an educational proton bakery our setup, as illustrated in Figure 2, included ingredients like ready-made cookies from the grocery store (mainly for hygiene reasons), quark-labelled multicoloured button-shaped chocolates (usually suggesting $m$-quarks), a few bottles of icing prepared on the spot, as well as some accompanying material [7-9].

'Proton Cookies' is not only eye- but also palate-catching and is also well suited to work as a queuing entertainment activity. 


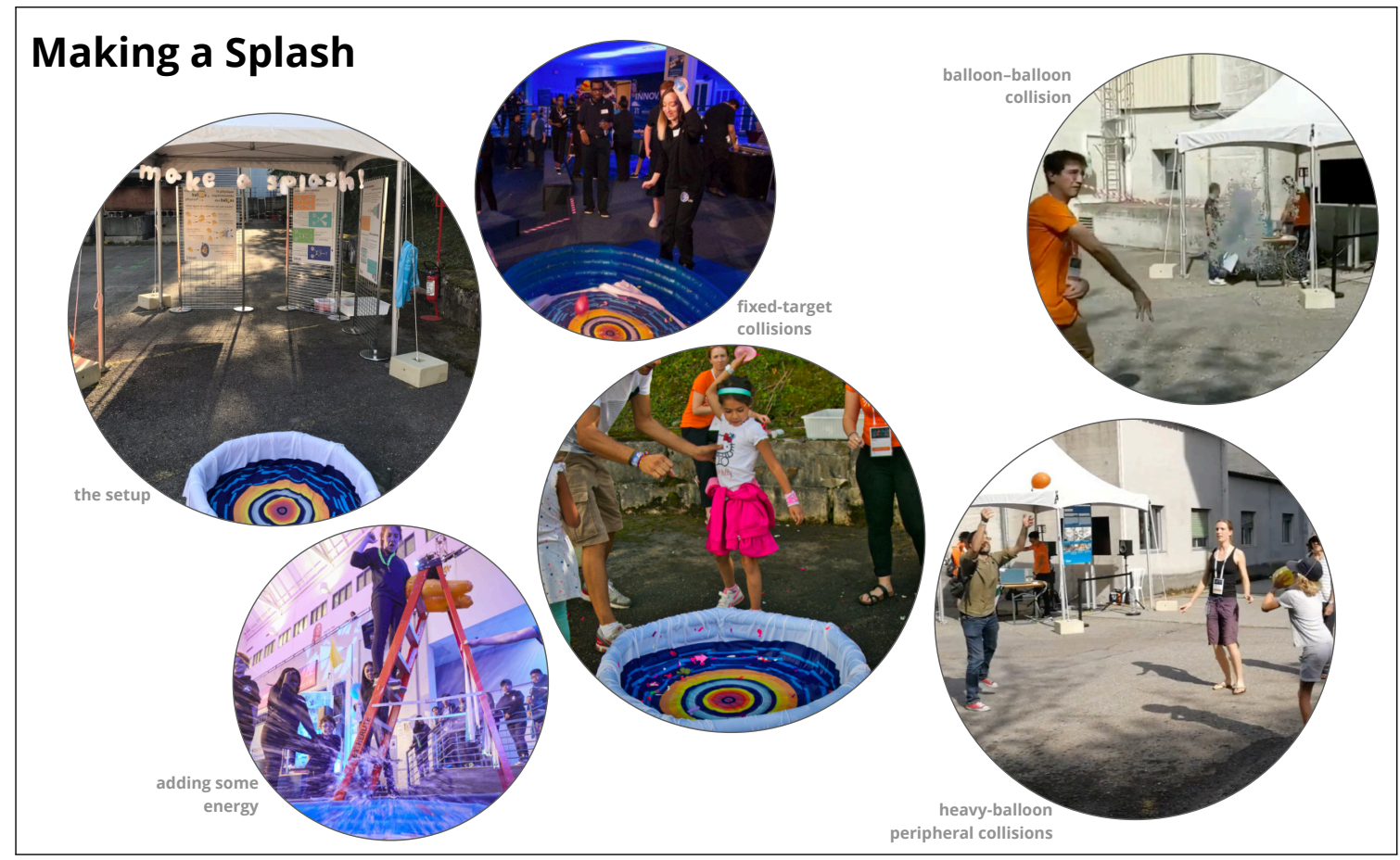

Figure 1: Some impressions of the 'Making a Splash' activity during the 2019 CERN Open Days and at TRIUMF.

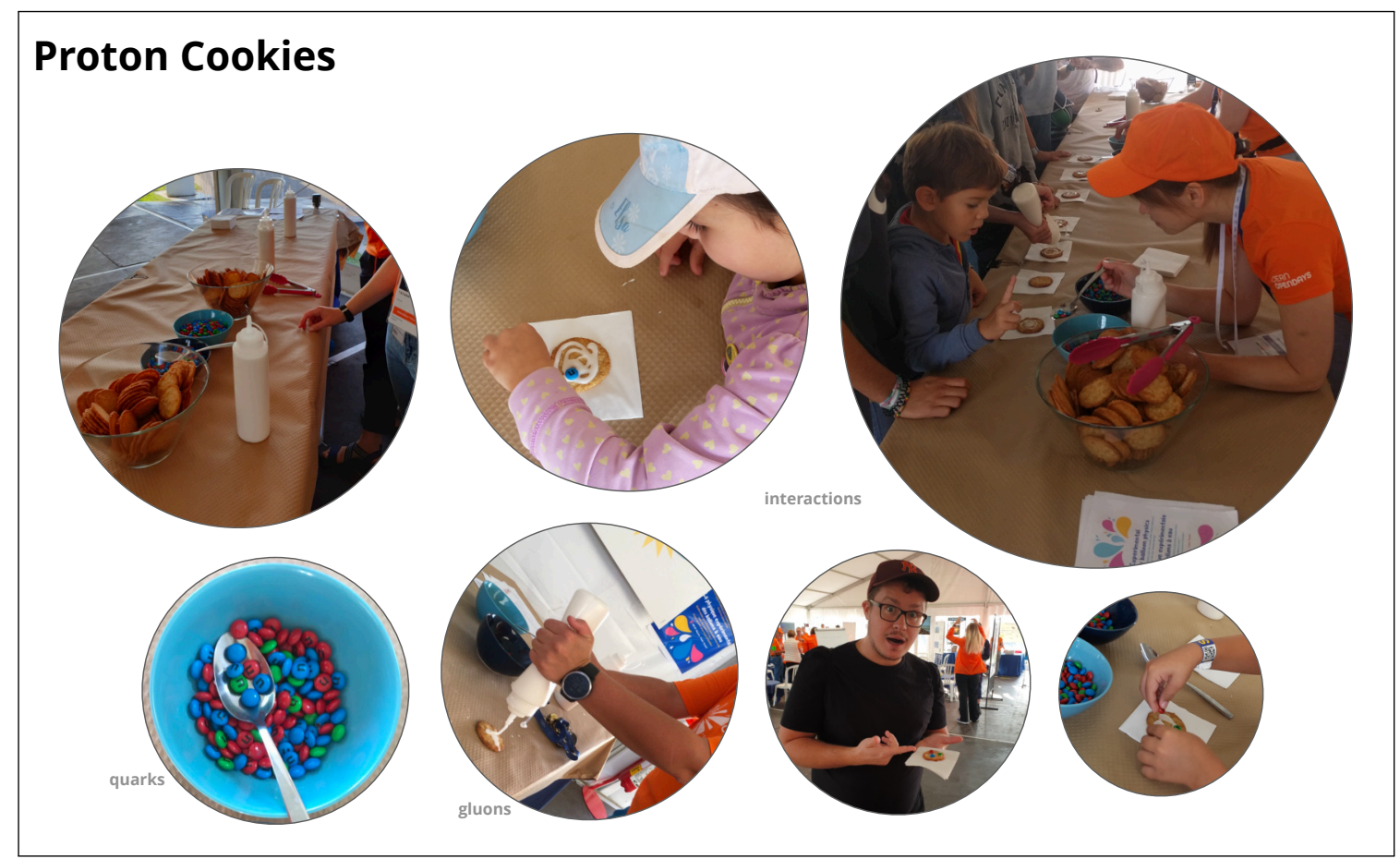

Figure 2: Some impressions of the 'Proton Cookies' activity during the 2019 CERN Open Days. 


\section{Build Your Own Particle Detector}

A long-standing and successful activity, already carried out at over 20 different events in eight places since 2013 and including more than 1300 registered participants, my 'Build Your Own Particle Detector' [10] activity invites visitors to build what they think a 'particle detector' does/should look like, using LEGO pieces, and register their designs in a competition. While constructing their experiments, participants and their companions can learn about the parts and purposes of real particle detectors, the challenges of design and construction (both LEGO and real experiments), the scale of particle detectors and the reasoning behind it, and more.

Running the 'Build Your Own Particle Detector', as illustrated in Figure 3, requires a few kilograms of (more or less) random LEGO pieces, posters and screens to inspire visitors with detector images, a camera and a computer, some prizes, tickets and a website to host the competition (the latter two - plus additional material - already available at Ref. [10]).

\section{Particle Twister}

'Particle Twister' - a particle-physics game with a twist [11] - invites visitors to combine physical and physics education in a remake of the original party game. While playing the game, both participants and the audience, can learn about the ingredients of the Standard Model of particle physics, interactions, quantum numbers, and many more aspects of high-energy physics.

Everything required to host an educational game of 'Particle Twister' - the playing board, a custom-designed spinner, playing-card templates for more involved game modes and more (particle-

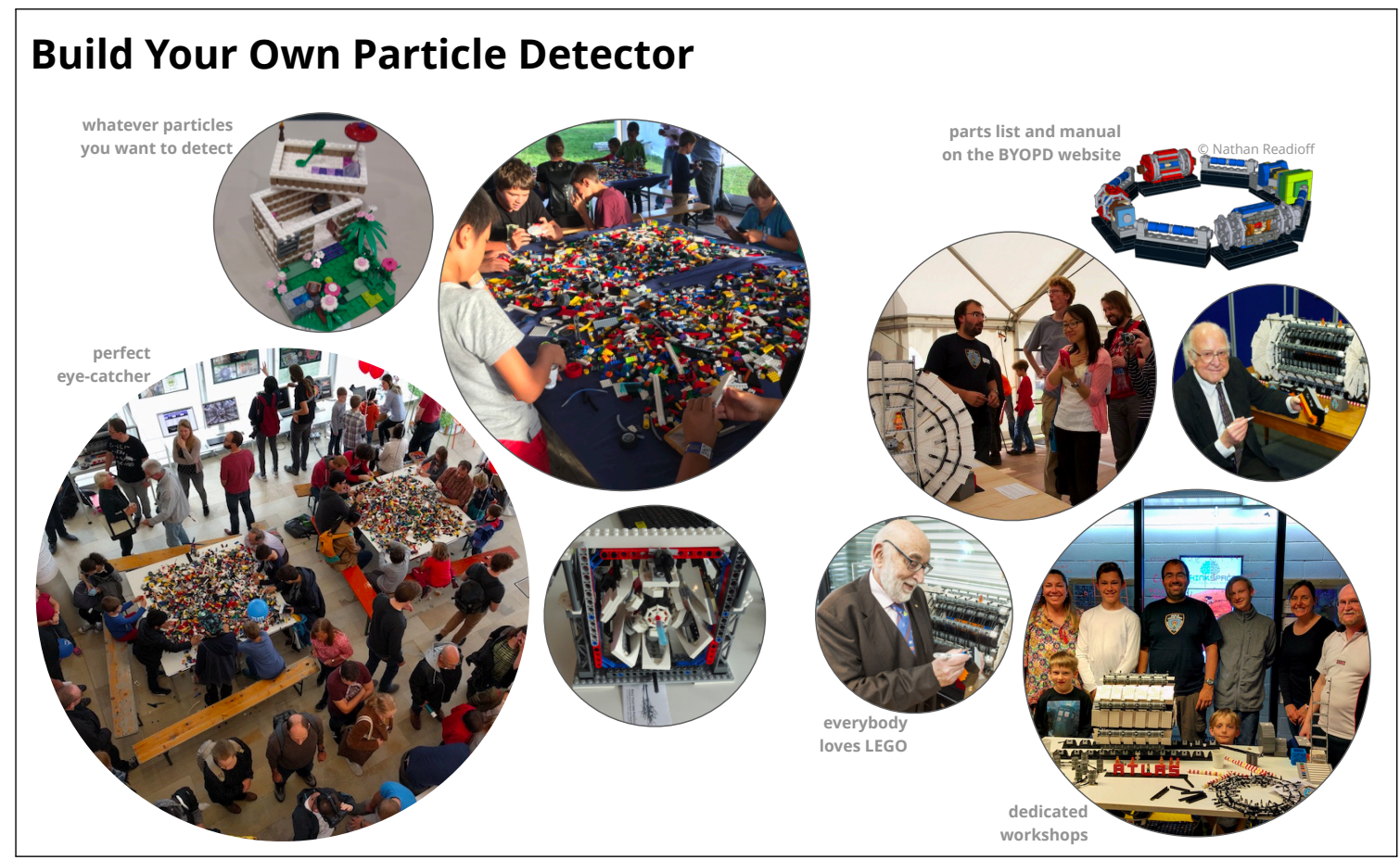

Figure 3: Some impressions of the 'Build Your Own Particle Detector' activity during some of the many events since 2013 . 


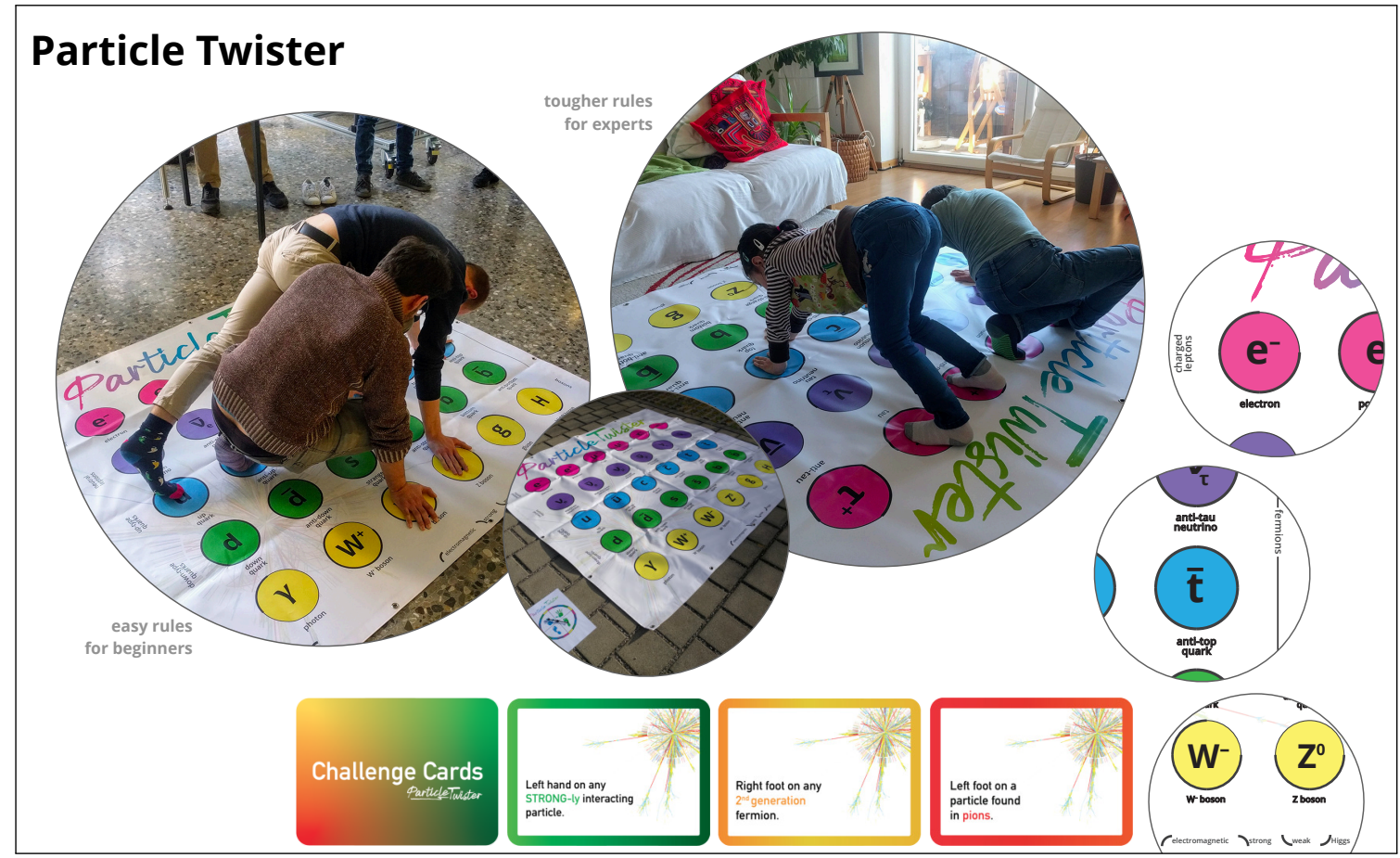

Figure 4: Some impressions of the 'Particle Twister' activity and some of the available material.

physics) experienced participants - is available at Ref. [11]. Figure 4 illustrates use cases, the board and some of the playing cards ... but remember to stretch before exercising.

\section{References}

[1] ATLAS Collaboration; The public website of the ATLAS Collaboration; https: //atlas.cern/

[2] ATLAS Collaboration; The ATLAS Experiment at the CERN Large Hadron Collider; JINST 3 (2008) S08003

[3] ATLAS Collaboration; Making a Splash Poster 1; https://cds . cern. ch/record/2724673

[4] ATLAS Collaboration; Making a Splash Poster 2; https://cds . cern. ch/record/2724675

[5] ATLAS Collaboration; Making a Splash Poster 3; https ://cds . cern. ch/record/2724677

[6] K. Leney, K. Tschann-Grimm; Physics Cakes; https://twitter . com/physicscakes

[7] ATLAS Collaboration / CERN; Proton Cookies Poster 1; https : //cds . cern. ch/record/2698601

[8] ATLAS Collaboration / CERN; Proton Cookies Poster 2; https: //cds . cern. ch/record/2698604

[9] ATLAS Collaboration / CERN; Proton Cookies Poster 3; https: //cds . cern. ch/record/2698952

[10] S. Mehlhase; Build Your Own Particle Detector; https: //build-your-own-particle-detector .org/

[11] K. Anthony, S. Mehlhase; Particle Twister; http://particle-twister.web.cern.ch/ 